\title{
Are nurse-led chemotherapy clinics really nurse-led? An ethnographic study
}

\begin{abstract}
Background: The number of patients requiring ambulatory chemotherapy is increasing year on year, creating problems with capacity in outpatient clinics and chemotherapy units. Although nurse-led chemotherapy clinics have been set up to address this, there is a lack of evaluation of their effectiveness. Despite a rapid expansion in the development of nursing roles and responsibilities in oncology, there is little understanding of the operational aspects of nurses' roles in nurse-led clinics.
\end{abstract} Objectives: To explore nurses' roles within nurse-led chemotherapy clinics.

Design: $\quad$ A focused ethnographic study of nurses' roles in nurse-led chemotherapy clinics, including semi-structured interviews with nurses.

Settings: Four chemotherapy units / cancer centres in the UK Participants: Purposive sampling was used to select four cancer centres / units in different geographical areas within the UK operating nurse-led chemotherapy clinics. Participants were 13 nurses working within nurse-led chemotherapy clinics at the chosen locations.

Methods: Non-participant observation of nurse-led chemotherapy clinics, semistructured interviews with nurse participants, review of clinic protocols and associated documentation.

Results: 61 nurse-patient consultations were observed with 13 nurses; of these 13, interviews were conducted with 11 nurses. Despite similarities in clinical skills training and prescribing, there were great disparities between clinics run by chemotherapy nurses and those run by advanced nurse practitioners. This included the number of patients seen within each clinic, operational aspects, nurses' autonomy, scope of practice and clinical decision-making abilities. The differences highlighted four different levels of nurse-led chemotherapy clinics, based on nurses' autonomy and scope of clinical practice. However, this was heavily influenced by medical consultants. Several nurses perceived they were undertaking holistic assessments, however they were using medical models / consultation styles, indicating medicalization of nurses' roles.

Conclusions: Four different levels of nurse-led chemotherapy clinics were identified, illustrating disparities in nurses' roles. Although clinics are run by nurses they are 
often controlled by medical consultants, which can reduce nurses' autonomy and negatively impact on patient care.

\section{Keywords}

Advanced nursing practice, Chemotherapy, Cancer, Nurse-led clinics, Nurse's roles

\section{Introduction}

Influenced by the European working time directive (Goddard et al 2010) there has been a rapid growth in nurse-led models of care and hybrid roles, such as advanced nurse practitioners which bridge the gap between nursing and medicine. However, in many cases this places greater emphasis on clinical / medical tasks than nursing care. Within oncology a rapid increase in the number and range of nurse-led clinics over the past ten years reflects developmental opportunities from UK cancer policies (NAO 2001, DH 2007, 2008, 2009, NCAG 2009) and changes to professional regulations. Changing legislation for independent nurse prescribing (DH 2006, Stenner and Courtenay 2008a, 2008b) has enabled UK nurses to prescribe medication independently (DH 2012, 2006, Courtney et al 2007), which has played a key role in developing nurse-led clinics.

In many countries, the majority of nurse-led clinics are set up to provide routine followup. This has enabled comparisons between nurse-led and medical clinics (Lee et al 2011, Strand et al 2011, Beaver et al 2010, Seibeck and Peterson 2009, Wells et al 2008). However, where studies focused on therapeutic relationships within nurse-led clinics this showed that nurse-led services can provide additional benefits for patients during radiotherapy (Wells et al 2008, Faithfull and Hunt 2005). Several studies identify nurse consultations to be longer than doctors (Strand et al 2011, Wells et al 2008, Allinson et al 2004, Baildam et al 2004, Campbell et al 1999); in most cases nurse-led clinics are deliberately set up to give patients at least twice as much time as medical clinics. The importance of continuity is also highlighted within a survey of 962 patients attending nurse-led clinics in Sweden, where $>90 \%$ of patients valued the continuity of seeing the same nurse at each visit (Berglund et al 2015). However, this did not include chemotherapy clinics. There is also strong evidence of greater patient-centred care when nurses take over whole episodes of care and address psychosocial issues or utilise nursing interventions (Corner et al 2002, Moore et al 2002). This highlights the 
importance of focusing on patients' needs and priorities for care within nurse-led clinics, rather than simply adopting medical models incorporating doctor-nurse substitution.

The number of patients requiring ambulatory chemotherapy is increasing year on year, placing greater burden on outpatient departments which are struggling to cope (Lennan et al 2012, Wiseman et al 2005), however there is little research on nurseled chemotherapy clinics. Endorsement of nurse-led chemotherapy by the National Chemotherapy Advisory Group (NCAG 2009) was an important landmark in promoting nurse-led chemotherapy services; however, this was ambiguous since it primarily referred to chemotherapy administration. Although nurses undertake chemotherapy administration in many countries (Wiseman et al 2005), the clinical management of patients undergoing a course of chemotherapy treatment is mainly undertaken by doctors. In the UK, some of this responsibility has devolved to senior nurses, including prescribing chemotherapy, assessing treatment toxicities and tolerability of treatment, within 'nurse-led' clinics (Lennan et al 2012).

Previous research indicates high patient satisfaction with chemotherapy nursing care, strongly influenced by interpersonal skills, including empathy, towards patients (Sitzia and Wood 1998). However, several studies indicate that chemotherapy nurses underestimated patients' symptoms (Mulders et al 2008, Sitzia and Wood 1998, Tanghe et al 1998), over-estimate patients' ability to cope (Sitzia and Wood 1998 ), and fail to identify $80 \%$ of patients' concerns during chemotherapy (Farrell et al 2005). The quality of nursing care in ambulatory chemotherapy is generally regarded as variable within the UK (National Chemotherapy Advisory Group 2009, Mort et al 2008), and evidence from several countries suggest this is a worldwide issue (Ekwall et al 2011, Hjorlifsdottir et al 2010, Arora 2009, Weingart et al 2007).

Despite an increased number of nurses becoming involved in the assessment / clinical management of patients during chemotherapy, there is little research evidence of safe care within nurse-led ambulatory chemotherapy, and no current evidence evaluating nurses' roles / operational practices (Farrell and Lennan 2013).

\section{Materials and methods}

This work has been carried out in accordance with The Code of Ethics of the World Medical Association (Declaration of Helsinki) for experiments involving humans 
(WMA 2016). Ethical approval was granted by the NHS Research Ethics Committee (REC) (reference $11 / \mathrm{NW} / 0240$ ) to undertake this multicentre study at different hospital locations. In addition, local Research and Development (R\&D) approvals were obtained from four hospitals in England prior to starting the study in that location. Sponsorship, indemnity and university ethics approval was also obtained from The University of Manchester.

\section{$\underline{\text { Aims }}$}

This study aimed to explore nurses' roles within nurse-led chemotherapy clinics and understand how the clinics operated in practice in order to answer the following research questions:

1. What are nurses' roles within nurse-led chemotherapy clinics?

2. What factors affect nurses' autonomy within nurse-led chemotherapy clinics?

\section{Research design}

Given the complexities for nurses' roles within nurse-led chemotherapy clinics observations and interviews were essential to achieve the required depth of understanding. Ethnography was chosen since its core focus is describing cultural behaviour (Shwandt 2007), which provides increased understanding of people's experiences within their local context (Roper and Shapira 2000, Fetterman 2010). Nurse researchers have used ethnography to explore issues influencing nursing practice (Streubert-Speziale and Rinaldi Carpenter 2007) and specific aspects of people's culture and experiences (Erickson 2011), including everyday interactions within the context of wider healthcare and organisational cultures (Savage 2006).

Focused ethnography is considered a useful method to understand specific aspects of peoples' lives (Cruz and Higginbottom 2013, Knoblauch 2005), often with a small group of people in a specific context (Roper and Shapira 2000). This approach can increase understanding of shared behaviours/ experiences within a specific group (Richards and Morse 2007), providing increased understanding of complex issues (Roper and Shapira 2000). This has particular merits for nurse researchers who aim to focus on nursing as a profession, inter-professional relationships / experiences and issues within their workplace environment (Cruz and Higginbottom 2013), therefore seemed a good approach for this study. 


\section{Sample and settings}

The setting for this study was nurse-led chemotherapy clinics in the UK. Purposive sampling strategies were used to target participants with specific knowledge / experience on the chosen topic (Higginbottom et al 2013). Four hospitals were chosen based on their geographical location to compare chemotherapy services in cancer units/centres at urban and rural locations. Nurses involved in nurse-led chemotherapy clinics at the four hospitals were invited to participate in the study.

The nurse-led clinics were chosen since they represented a 'typical day' in terms of the number of patients seen, the types of chemotherapy regimens and other systemic infusions given. Some hospitals had clinics for specific cancer groups on certain days, which the observations covered; other nurse-led clinics had two or three sessions per week, therefore observations covered several sessions / clinics on different days, which resulted in approximately 12 days of observation across the four sites.

Written informed consent was obtained from all nurse participants prior to the study requesting permission to observe their nurse-led chemotherapy clinics for two days and be interviewed by a researcher. Patients received an information leaflet on arrival at the chemotherapy unit, and information posters were also displayed. Patients gave verbal consent before their consultation in the nurse-led clinic and researcher observation; they were informed that they could decline observation or aske the researcher to leave at any point.

\section{Data collection}

In ethnography, the three data collection strategies: participant observation interviews and examination of relevant documents are considered essential (Cruz and Higginbottom 2013, Fetterman 2010). Interviews are considered crucial in validating observational data and incorporating participants' feelings/perceptions (Higginbottom et al 2013, Fetterman 2010). Examining relevant documents, such as policies and procedures, can also help validate data from observation/interviews (Roper and Shapira 2000). This triangulation of data collection strategies enables multiple comparisons from data sources to confirm the findings (Mueke 1994), and may also reveal covert dimensions that may not be articulated by participants (Fetterman 2010). 
This study included non-participant observation of nurse-patient consultations, which were audio-recorded. Numerical codes were assigned to each patient /nurse to maintain confidentiality/anonymity throughout. Nurses were interviewed in a private room after the first observation session; interviews were audio-recorded and an interview guide was used; questions included setting up nurse-led clinics, operational aspects, managerial support and training. A key advantage of observing nurses within their clinical environment would be greater understanding of the context for each nurse-led chemotherapy clinic, operational aspects of nurses' roles and to observe nurses' interactions with patients and colleagues. This valuable information could not be obtained from interviews alone (Roper and Shapira 2000). Semi-structured interviews with nurses were used to validate observations, clarify aspects of practice and understand nurses' perceptions (Roper and Shapira 2000, Kleiman 2004); studying documentation provided additional information about operational aspects of each nurseled chemotherapy clinic. The combination of observation, interviews and documentation also aimed to check that what people say they do is the same as what they actually do (Mulhall, 2003).

\section{Data analysis}

Data analysis included the following steps to address focused research questions:

- $\quad$ Coding (descriptive labelling)

- $\quad$ Sorting (establishing patterns)

- $\quad$ Constant comparison (identifying outliers)

- Developing constructs and theories

- $\quad$ Memoing (including reflective data) (Higginbottom et al 2013).

Data analysis was inductive and started during data collection, reflexively discovering themes from the data and continually reviewing the recordings and notes of what participants said and did (Roper and Shapira 2000). All audio data was transcribed prior to data analysis. First level coding included applying descriptive labels to chunks of text, which summarized the content and was later combined into broader categories. Microsoft WORD and Excel were used to create summaries, flowcharts, diagrams and visual displays to facilitate data analysis and comparisons of nurses' roles and operational issues within and between cancer centres/units.

\section{Results}


Thirteen nurse participants were observed within nurse-led chemotherapy clinics: two were nurse consultants, three advanced nurse practitioners (ANP) and eight chemotherapy nurses. All had received training in clinical examination skills and nonmedical prescribing. Twelve days of observation was undertaken at four hospitals over six months. This included 61 nurse-patient observations with 13 nurses; of these 13, interviews were conducted with 11 nurses. Nurse consultations ranged from 3-60 minutes (mean 20.26); interviews ranged from 12-47 minutes (mean 26.7).

\section{Drivers for nurse-led chemotherapy clinics}

The main driver for nurse-led clinics was the reduction in medical staff and increasing clinical demands. Clinics were set up by experienced nurses in three locations using protocols to guide clinical practice. Protocols included criteria for patient suitability for nurse-led clinics, the nature of assessments required and criteria for referral to doctors. In the fourth location protocols were not used since advanced nurse practitioners held nurse-led chemotherapy clinics for patients within one cancer group, and had similar clinical responsibilities to doctors.

The following findings address the aims of the study and answer the two research questions:

\section{What are nurses' roles within nurse-led chemotherapy clinics?}

There was a great deal of variability within nurses' roles and responsibilities at nurse-led chemotherapy clinics in the four hospitals, including the scope of nurses' clinical practice. This was reflected by operational aspects of the nurse-led clinics, including the way clinics had been set up. Nurses at one location focused primarily on chemotherapy administration; other locations conducted separate nurse-patient chemotherapy reviews during treatment. These were described as 'closed door' consultations since they were in a private room; nurses assessed patients' progress during chemotherapy, including toxicities and blood results before prescribing the next cycle of chemotherapy and antiemetics.

Although all nurse participants had completed training in clinical examination skills and independent non-medical prescribing, observations revealed clear differences in their roles, responsibilities, and scope of clinical practice at each hospital. This highlighted 
disparities in nurses' autonomy, which appeared complex and influenced by several factors.

\section{What factors affect nurses' autonomy within nurse-led chemotherapy clinics?}

Several factors influenced nurses' autonomy within nurse-led chemotherapy clinics; the main factors included written protocols for nurse-led clinics, operational aspects, personal / professional issues, and nurse-doctor relationships.

\section{Written protocols for nurse-led clinics}

The selection of appropriate patients for nurse-led chemotherapy clinics is crucial however some protocols restricted the potential number of patients that could be seen in nurse-led clinics. For example, placing limitations on chemotherapy regimens resulted in very small numbers of patients in nurse-led clinics and negligible impact on patient numbers within medical clinics. The referral pathway outlined in protocols was not always followed; doctors were selective in referring patients to clinics run by chemotherapy nurses so patients eligible for nurse-led were often kept within medical clinics. Consequently, clinic numbers remained small and nurses faced criticism from medical consultants for this.

Independent nurse prescribing was the lynch pin to developing nurse-led clinics and autonomous clinical practice, however protocols for nurse-led chemotherapy clinics were often written by medical consultants who limited the range of medicines that nurses could prescribe independently. In contrast, protocols written jointly by nurses and medical consultants were less prescriptive.

\section{Operational aspects of nurse-led clinics}

At all four locations doctors directly controlled referrals to nurse-led chemotherapy clinics. Although nurses intended to increase patient numbers and expand their referral criteria, this was slow to materialise; capacity issues continued given small patient numbers in nurse-led clinics and limited referrals from doctors. This left nurses feeling frustrated; however instead of challenging doctors they adopted covert strategies to divert patients from the medical clinics into their nurse-led clinics. Nurses also felt used when doctors were inconsistent in referring patients, since referrals to nurse-led clinics; 
increased when doctors were on leave. In contrast, clinics run by ANPs had a high number of patients; however too many patients resulted in clinics running late and increased waiting times for patients, which placed additional pressure on nurses. Appointment slots in nurse-led clinics were also much longer than medical clinics, raising questions about viability, sustainability and cost-effectiveness.

\section{Personal / professional issues}

There were some disparities between proposed activities documented within protocols and clinic observations. For example, holistic assessments were recommended in protocols but not observed within nurse-led clinics, although nurses perceived their assessments were holistic. During observations nurses demonstrated a thorough knowledge of medicines and discussed this appropriately with patients, however chemotherapy nurses were unable to adjust the dose of chemotherapy and defer / stop treatment, which increased waiting time for patients. There were similar restrictions such as managing toxicities above grade 2 , and making decisions when blood counts exceeded set parameters. Problems also arose if patients were too complex for nurses to manage effectively and safely; for example, some chemotherapy nurses lacked detailed knowledge about oncology to answer questions about a patient's cancer and to give scan results. This contrasted sharply with the responsibilities and decision-making abilities of ANPs within their nurse-led clinics.

\section{$\underline{\text { Nurse-doctor relationships }}$}

Nurses' relationships with medical consultants appeared to have a clear impact on nurses' roles, their scope of practice and autonomy within nurse-led clinics. Nurses recognised the importance of developing good relationships with medical consultants and obtaining support from their hospital Trust, highlighting how this influenced patient referrals.

The study identified that specialist nurses (ANPs) appeared to have a stronger relationship with medical consultants, which developed from close working practices within a disease group. Consequently, ANPs were awarded a higher level of autonomy and clinical decision-making. In contrast, chemotherapy nurses worked with many different consultants, therefore had to rely on generic protocols to guide their practice. Although medical consultants generally accepted the expansion of nurses' roles and 
nurse-led clinics, there were tensions about clinic names and patient numbers, which suggest issues of 'ownership' and 'power' between nurses and medical consultants.

In one location, there was evidence of role reversal in that some ANPs were supervising and training medical registrars, and some registrars supported nurse-led chemotherapy clinics. This changes team dynamics and may create some resistance from junior doctors. However, at other locations the position between consultants, registrars and chemotherapy nurses seemed more hierarchical with doctors controlling changes.

\section{Additional findings}

Two additional findings emerged from the data and appear to be key findings from the study:

\section{Four levels of nurse-led chemotherapy clinics}

The findings indicate that nurse-led chemotherapy clinics consist of four different levels, depending on nurses' skills, clinical responsibilities and level of autonomy. This description aims to provide a basic structure and definition for nurse-led chemotherapy clinics, and has implications for clinical practice, professional development and service delivery (table 3).

\section{Advanced nursing practice or medicalization of nurses' roles?}

During the initial development of nurse-led chemotherapy clinics, nurses used written checklists to assess chemotherapy toxicities, however this practice decreased as nurses' confidence improved. Nevertheless, this influenced nurses' consultations with patients since they often used a 'checklist' approach to communication, which placed greater emphasis on the physical effects and practical aspects of chemotherapy, with less emphasis on psycho-social concerns. Despite this, nurses perceived they were using a holistic approach.

Several nurses perceived that medicalization of nursing roles represented advanced nursing practice, and considered a 'skills' set' to be essential. Several nurses described a collection of clinical skills as essential criteria for nurse-led clinics, including nonmedical prescribing and clinical examination skills, although some nurses failed to use such skills in clinical practice. However, some nurses highlighted the importance of nursing within advanced practice, rather than simply doctor-nurse substitution. 
Nevertheless, nurses chose to use medical models and medical consultation styles in their own clinical practice.

Expanding nurses' skills to take on medical responsibilities brought concerns that medical colleagues would become deskilled. This appeared proportional to the degree of medicalization in nurses' roles and responsibilities. At one location nurses perceived that their roles had the potential to deskill consultants, whereas other locations perceived their roles would deskill junior medical staff, including registrars.

It seems that as nurses increase their knowledge, advance their clinical skills and take on medical responsibilities, this changes the power dynamics within multi-disciplinary teams. Blurring the boundaries of professional roles may create tensions between nurses, junior doctors and medical consultants, however, nurses' autonomy and beliefs may also provide significant influences.

\section{Summary of key findings}

Despite similarities in clinical skills training and prescribing, there were great disparities between clinics run by chemotherapy nurses and those run by ANPs. This included the number of patients seen within each clinic, operational aspects, nurses' autonomy, scope of practice and clinical decision-making abilities. These differences highlighted four different levels of nurse-led chemotherapy clinics, based on nurses' autonomy and scope of clinical practice. However, this was heavily influenced by medical consultants. Several nurses perceived they were undertaking holistic assessments, however they were using medical models / consultation styles, indicating the medicalization of nurses' roles.

\section{Discussion}

The theme of power within nurse-led chemotherapy clinics was unexpected and complex. The data identified strong influences by medical consultants over nurse-led chemotherapy clinics in the way clinics were set up, how they were run and within the scope of nurses' clinical practice. Professional relationships with medical colleagues are vital to the success of nurse-led clinics, and medical consultants have the power to make or break nurse-led clinics through patient referrals and also their professional influence / control. There is strong evidence that inter-professional teamwork can affect 
the quality of patient care in oncology (Friese and Manojlovich 2012, Ponte et al 2010, Manojlovich 2010); supportive relationships and good communication are important team attributes that result in superior team performances (Gittell et al 2000). Within this, supportive relationships were viewed as having shared knowledge / goals and mutual respect, whilst good communication included timely, frequent and problem-solving communication (Lee et al 2014).

Previous research highlights how power differences and professional logistics may influence inter-professional collaboration (San Martin-Rodriguez et al 2005). An observational study of oncology outpatient clinics in Ontario identified high levels of collaboration and interaction between nurses and physicians, although this may be influenced by environmental and organisational factors (Lee et al 2014). Power struggles and gender/class divisions were also identified in an ethnographic study on a paediatric critical care unit, which affected nurses' abilities to undertake research activities (Scott and Pollock 2008).

Membership of a group is complex and the insider/outsider roles are not absolute (Merton 1972, Naples 1996); being an insider within the same profession and organisation as participants can be challenging (Simmons 2007). Roberts (2007) suggests that insider knowledge can be beneficial in producing greater understanding of the culture, and previous familiarity between researcher and participants can also promote more honesty in participants' responses than if they were strangers (Bonner and Tolhurst 2002). However, participants may exercise selectivity in disclosing information to researchers (Alvesson 2003, Roberts 2007). This seems particularly important where researchers may have in-depth knowledge or personal experience of the topic being studied (Cruz and Higginbottom 2013). Often focused ethnography is carried out within the researcher's own workplace, therefore the concept of reflexivity is important during data analysis (Higginbottom et al 2013).

As an advanced nurse practitioner in oncology I was an insider to the profession with knowledge and experience of working at a high level of clinical practice, including independent prescribing. I was an insider to the setting of chemotherapy nurse-led clinics, with experience of setting up and running my own clinics independently, and an insider within one organisation but an outsider to three organisations. I observed some nurses who I knew professionally, and others who I had not previously met. My insider 
experience facilitated access to organisations and participants, which may have enhanced the level of trust from participants (Fetterman 1998). It may also have increased nurses' openness during observations and interviews. However, I have no evidence of this during data collection, and familiarity with some participants also brought its own challenges during data collection and analysis.

The data revealed that although nurses ran their own nurse-led clinics, all nurses remained dependent on medical colleagues to some extent, irrespective of the degree of collaboration. Even where nurses had a good relationship with consultants and greater responsibilities during a patient's chemotherapy, patients will still 'belong' to medical consultants. Nurse-led chemotherapy clinics rely on referrals by medical staff, therefore if the referrals stop the nurse-led clinic will fail. This process is heavily influenced by professional relationships between medical consultants and nurses; however, the findings from this study identify more frequent issues where nurses share chemotherapy management with medical staff. This highlights the potential vulnerability of nurses who undertake nurse-led clinics, in that no matter how autonomous the role is perceived to be, or how skilled nurses are, nurses and their nurse-led clinics are dependent on medical staff.

Determining the appropriate number of patients for nurse-led clinics is challenging; if numbers are too low, nurses may face criticism from medical colleagues, and if numbers are too high this may leave nurses feeling overwhelmed. The findings demonstrate the difficulties of developing ad hoc nurse-led services; coupled with a lack of research, no forward planning, and insufficient resources, reflecting previous discussions of challenges within the NHS (Lennan and McPhelim, 2012). Questions regarding the viability of nurse-led clinics will be raised where patient numbers are small, particularly given the salary costs of specialist and chemotherapy nurses. This is important in the current financial climate given the vulnerability of specialist nurse roles and requirements to provide value for money (Kelly and Trevatt, 2006; Barlow and Jackson, 2007).

Considering nurse-led chemotherapy clinics as a collective term is inappropriate, since the findings of this study identify four different levels of nurse-led practice within nurseled chemotherapy clinics. This finding is novel, and will be valuable for patients, health professionals, commissioners and policy-makers in defining chemotherapy services. 
The four different levels of nurse-led chemotherapy clinics identified in this study highlight the complexities within clinics and suggest the use of umbrella terms is inappropriate. Increased precision is required when describing nurse-led clinics to ensure accuracy in perceived autonomy, scope of clinical practice and patient care. This has implications for service delivery, hospital managers, commissioners and policymakers to ensure clinical services are developed appropriately.

Within the current NHS there is increasing pressure on chemotherapy services from rising numbers of patients needing chemotherapy. Whilst nurse-led chemotherapy clinics may seem to provide the quick fix from a government perspective, greater investment is required if this is to be a sustainable solution, given the complexities involved. It seems foolhardy to make recommendations for more nurse-led chemotherapy clinics in the absence of transparent definitions, particularly given the lack of research and disparate levels of clinical practice.

The development of protocols to guide the nurse-led clinics identifies considerable influence by medical staff, with examples of biomedical models of care, prescriptive clinical management and restrictive nursing practice. Whilst protocols may be beneficial to clarify operational processes, 'nursing assessments' and psychosocial aspects of care during chemotherapy were not addressed. This contrasts with the position statement by the UK Oncology Nursing Society (UKONS), which recommends that nurse-led clinics should not duplicate a medical model, but provide additional benefits for patients in terms of holistic care and family support (Lennan et al, 2012). Furthermore, the National Cancer Action Team (2012) recommend that chemotherapy nurses should also ensure that patients' "wider holistic needs" are addressed within nursing assessments and care (NCAT, 2012). Clearly this was not evident within this study and it raises concerns that some nurses perceived their practice to be holistic when this did not match independent observations of their actual practice.

Nurses' job titles were not a reliable indicator of their roles and responsibilities. There was individual variability within nurse-led chemotherapy clinics and clear differences in the way specialist nurses and chemotherapy nurses ran their nurse-led chemotherapy clinics. There is evidence in the literature where greater autonomy enabled nurses to be more flexible and creative with nurse-led clinics (Faithfull et al, 2001; Wells et al, 2008), rather than simply using a medical model with doctor-nurse substitution (Campbell et al, 
1999). However, within this study nurse-led chemotherapy clinics adopted a medical model, with no evidence of alternative nurse-led models of care.

Some nurses received training in clinical examination but did not use their skills in clinical practice, influenced by lack of confidence, which suggests a waste of time and resources. However, this contrasts with nurse prescribing, where limitations were primarily caused by restrictions by medical staff, rather than nurses' lack of confidence. Changes to legislation for independent nurse prescribing have had the greatest impact on nurse-led chemotherapy clinics by enabling nurses to prescribe supportive medication for chemotherapy (Farrell and Lennan, 2013; Lennan et al, 2012). The UK Oncology Nursing Society (Lennan et al, 2012) proposes that nurse prescribing within the clinic is the 'gold standard' for nurse-led chemotherapy review clinics.

Willard and Luker (2007) identified that acceptance by doctors was the main problem facing cancer clinical nurse specialists, which may impair nurses' ability to provide supportive care to patients. Acceptance was crucial since nurses relied on referrals from medical staff, and nurses devised a variety of strategies to increase acceptance, including building relationships with senior doctors, avoiding criticism and using a gentle approach to change (Willard and Luker, 2007).

\section{Strengths and limitations}

The strength of this study lies in the use of an ethnographic observational approach. This enabled a comparison of what nurses say they do (interviews) and what they actually do (observations) in clinical practice, which revealed disparities. If this study had relied on interviews only the results would have indicated that nurses were undertaking holistic assessments, however this was not demonstrated during nursepatient observations. Similarly, nurses expressed confidence in using clinical examination skills during interviews, but observations revealed a lack of confidence when examining patients in their clinics. Therefore, observations provided greater knowledge of nurses' roles and scope of practice.

The main limitation of this study was the restriction to nurse-led chemotherapy clinics in the UK, although steps were taken to ensure coverage of different geographical locations and include both large cancer centres and smaller cancer units. However, the 
inclusion of nurses from other countries would have added a greater international perspective and increased the transferability of the findings.

\section{Conclusions}

The findings from this study highlight several issues, illustrating power tensions between nursing and medical staff that have, in some cases, restricted nurses' scope of clinical practice in nurse-led chemotherapy clinics.

Most chemotherapy nurses did not undertake any clinical examinations, which has implications for future training opportunities, particularly in the current financial climate. The identification of four different levels of nurse-led chemotherapy clinics is a key finding, which has implications for nurses, patients, and chemotherapy services. Implementation of this framework would provide national standards to benchmark nurse-led chemotherapy services in the UK, although it may have relevance for other countries. The framework would also facilitate the development of competencies and training across each of the four levels, which may lead to national recognition of skills and competencies within different levels of clinical practice. Further development of a chemotherapy skills passport would also enable nurses to transfer their skills to different organisations within the UK, saving time and resources for training and providing greater benefits for patients and clinical services.

\section{References}

Allinson V. (2004). Breast cancer: evaluation of a nurse-led family history clinic. Journal of Clinical Nursing. 13:765-766.

Alvesson M (2003). Methodology for close up studies - struggling with closeness. Higher Education. 46(2): 167-193.

Arora N (2009). Importance of patient-centred care in enhancing patient well-being: a cancer survivor's perspective. Quality of Life Research. 18:1-4.

Baildam A, Keeling F, Thompson L, Bundred N, Hopwood P. (2004). Nurse-led surgical follow-up clinics for women treated for breast cancer - a randomised controlled trial. European Journal of Cancer S136-7

Barlow H, Jackson T. (2007). Preparing for leadership: the ability to lead emerges from a variety of skills and experiences at the senior level of nursing. Cancer Nursing Practice. 6 (3): 14-17. 
Beaver K, Williamson S, Chalmers K. (2010). Telephone follow up after treatment for breast cancer: views and experiences of patients and specialist breast care nurses. Journal of Clinical Nursing. 19(19-20): 2916-24

Berglund CB, Gustafsson E, Johannsson H, Bergenmar M (2015). Nurse-led outpatient clinics in oncology care - patient satisfaction, information and continuity of care. European Journal of Oncology Nursing. 19:724-730.

Bonner A, Tolhurst G (2002). Insider-outsider perspectives of participant observation. Nurse Researcher. 9(4): 7-19.

Campbell J, German L, Lane C (1999). Radiotherapy outpatient review: a nurse-led clinic. Nursing Standard.13(22):39-44

Corner J, Moore S, Haviland J et al (2002). Nurse-led follow up and conventional medical follow up in management of patients with lung cancer: randomised trial. BMJ. 325: 1145-47

Courtenay M, Carey N, Burke J. (2007). Independent, extended and supplementary nurse prescribing in the UK: a national questionnaire survey. International Journal of Nursing Studies. 44(7): 1093-1101.

Cruz EV, Higginbottom G (2013). The use of focused ethnography in nursing research. Nurse Researcher. 20(4): 36-43.

Department of Health. (2012). Nurse and pharmacist independent prescribing changes announced. https://www.gov.uk/government/news/nurse-and-pharmacistindependent-prescribing-changes-announced [last accessed 19.10.2013]

Department of Health. (2009). Impact assessment of national chemotherapy advisory group recommendations. http//tinyurl.com/8a7pfyq [accessed 25.03.2012]

Department of Health. (2008). High quality care for all: NHS Next Stage Review. London, DH

Department of Health. (2007). The Cancer Reform Strategy. DH, London.

Department of Health. (2006). Improving Patients' Access to Medicines: A guide to implementing nurse and pharmacist independent prescribing within the NHS in England. London, DH

Ekwall E, Ternestedt BM, Sorbe B, Graneheim UH (2011). Patients' perceptions of communication with the health care team during chemotherapy for the first recurrence of ovarian cancer. European Journal of Oncology Nursing. 15:53-58.

Erikson $F$ (2011). A history of qualitative inquiry in social and educational research. In: Denzin NK, Lincoln Y (eds). The SAGE handbook of qualitative research. Fourth edition. Sage Publications, Thousand Oaks, CA

Faithfull S, Hunt G. (2005). Exploring nursing values in the development of a nurseled service. Nursing Ethics. 12(5): 440-52. 
Faithfull S, Corner J, Meyer L, Huddart R, Dearnaley D. (2001). Evaluation of a nurse-led follow up for patients undergoing pelvic radiotherapy. British Journal of Cancer. 85(12): 1853-1864.

Farrell C, Lennan E. (2013). Nurse-led chemotherapy clinics: Issues for the prescriber. Nurse Prescribing. 11(7): 561-566.

Farrell C, Heaven C, Beaver K, Maguire P (2005). Identifying the concerns of women undergoing chemotherapy. Patient Education and Counseling 56 (1): $72-7$

Fetterman DM (2010). Ethnography Step by Step. Third Edition. Sage Publications, Thousand Oaks, CA.

Fetterman DM (1998). Ethnography step by step. Sage Publications, Thousand Oaks, CA.

Friese CR, Manojlovich M (2012). Nurse-physician relationships in ambulatory oncology settings. Journal of Nursing Scholarship. 44:258-265.

Gittell JH, Fairfield KM, Bierbaum B, Head W, Jackson R, Kelly M et al (2000). Impact of relational coordination on quality of care, post operative pain and functioning, and length of stay: a nine hospital study of surgical patients. Medical care. 38: 807-819.

Goddard A, Hodgson H, Newbery N, (2010) Impact of EWTD on patient: doctor rations and working practices for junior doctors in England and Wales. Clinical Medicine 10(4): 1-6

Higginbottom GMA, Pillay JJ, Boadu NY (2013). Guidance on performing focused ethnographies with an emphasis on healthcare research. The Qualitative Report 18(17): $1-16$.

Hjorlifsdottir E, Hallberg IR, Gunnarsdottir ED (2010). Satisfaction with care in oncology outpatient clinics: psychometric characteristics of the Icelandic EORTC INPATSAT32 version. Journal of Clinical Nursing. 19:1784-1794.

Kelly D, Trevatt P (2006). NHS finances. Cancer Nursing Practice 5(8), 14-18

Kleiman S (2004). What is the nature of nurse practitioners' lived experiences interacting with patients? J Am Acad Nurse Pract 16 (6): 263-9.

Knoblauch H (2005). Focused ethnography forum. Qualitative Social Research. 6(3):44.

Lee CT, Doran DM, Tourangeau AE, Fleshner NE (2014). Percdeived quality of interprofessional interactions between physicians and nurses in oncology outpatient clinics. European Journal of Oncology Nursing. 18:619-625.

Lee H, Lim Y, Yoo MS, Kim Y. (2011). Effects of a nurse-led cognitive behaviour therapy on fatigue and quality of life in patients with breast cancer undergoing radiotherapy: An exploratory study. Cancer Nursing. 34(6): 22-30 
Lennan E, Vidall C, Roe H, Jones P, Smith J, Farrell C (2012). Best practice in nurse-led chemotherapy review: a position statement from UKONS. ecancer 6:263 doi:10.3332/ecancer.2012.263

Lennan E, McPhelim J. (2012). A snapshot of innovation: chemotherapy delivery in the UK. British Journal of Nursing. 21(4): S12-15.

Manojlovich M (2010). Nurse/physician communication through a sense-making lens: shifting the paradigm to improve patient safety. Medical Care. 48:941-946.

Merton RK (1972). Insiders and outsiders: A chapter in the sociology of knowledge. American Journal of Sociology. 78(1): 9-47

Moore S, Corner J, Haviland J et al (2002). Nurse-led follow up and conventional medical follow up in management of patients with lung cancer: randomised trial. British Medical Journal. 325:1145.

Mort D, Lansdown M, Smith N, Protopapa K, Mason M. (2008). For Better, for Worse? A Review of the Care of Patients Who Died within 30 days of Receiving Systemic Anti-cancer Therapy (Report by the National Confidential Enquiry into Patient Outcome and Death). NCEPOD.

Mueke MA (1994). On the evaluation of ethnographies. In: JM Morse (Ed). Critical issues in qualitative research methods. Sage Publications, Thousand Oaks, CA.

Mulders M, Vingerhoets A, Breed W (2008). The impact of cancer and chemotherapy: perceptual similarities and differences between cancer patients, nurses and physicians. European Journal of Oncology Nursing. 12:97-102.

Mulhall A. (2003). In the field: notes on observation in qualitative research. Journal of Advanced Nursing. 41(3): 306-313.

Naples NA. (1996). The outsider phenomenon. In C. D. Smith \& W. Kornblum (Ed.), In the field: Readings on the field research experience (2nd ed., pp. 139-149). Westport, CT. Praeger.

National Audit Office (2001). Inpatient and outpatient waiting in the NHS: report by the comptroller and auditor general. Department of Health NAO. HC221.

National Cancer Action Team (2012). Quality in nursing: A census of the chemotherapy nursing workforce in England 2012. London, Department of Health.

National Chemotherapy Advisory Group (2009). Chemotherapy services in England: Ensuring quality and safety. A report from the National Chemotherapy advisory Group. Department of Health. London.

Ponte PR, Goss AH, Milliman-Richard YJ, Lacy K (2010). Interdisciplinary teamwork and collaboration: an essential element of a positive practice environment. Annual of Nursing Research. 28:159-189.

Richards L, Morse JM (2007). Readme first for a user's guide to qualitative methods. Second Edition. Sage Publications, Thousand Oaks, CA. 
Roberts D (2007). Ethnography and staying in your own nest. Nurse Researcher. 14(3):15-24.

Roper JM, Shapira J. (2000). Ethnography in nursing research. Sage, London.

San Martin-Rodriguez L, Beaulieu MD, D'Amour D, Ferrada-Videla M (2005). The determinents of successful collaboration: a review of theoretical and empirical studies. Journal of Interprofessional Care. 19(s1): 132-147.

Savage $J(2006)$. Ethnographic evidence: The value of applied ethnography in healthcare. Journal of Research in Nursing. 11: 383-393.

Seibaek L, Petersen LK (2009). Nurse-led rehabilitation after gynaecological cancer surgery: provisional results from a clinically controlled prospective questionnaire study. Supportive Care in Cancer. 17(5):601-5.

Schwandt TA (2007). The Sage dictionary of qualitative inquiry. Third Edition. Sage Publications, Thousand Oaks, CA.

Scott SD, Pollock $C$ (2008). The role of nursing unit culture in shaping research utilization behaviours. Research in Nursing and Health. 31(4): 298-309.

Simmons M (2007). Insider ethnography: tinker, tailor, researcher or spy? Nurse Researcher. 14(4): 7-17.

Sitzia J, Wood N (1998). Study of patient satisfaction with chemotherapy nursing care. European Journal of Oncology Nursing. 2(3): 142-153.

Stenner K, Courtenay M. (2008a). The role of inter-professionals relationships and support for nurse prescribing in acute and chronic pain. Journal of Advanced Nursing. 63(3): 276-83.

Stenner K, Courtenay M. (2008b). Benefits of nurse prescribing for patients in pain: nurses' views. Journal of Advanced Nursing. 63(1): 27-35.

Strand E, Nygren I, Bergkvist L, Smedh K (2011). Nurse or surgeon follow up after rectal cancer: A randomized trial. Colorectal Disease. 13(9):999-1003.

Streubert-Speziale HJ, Rinaldi Carpenter D (2007). Ethnography as a method. In: Streubert-Speziale HJ, Rinaldi Carpenter D (Eds). Qualitative Research in Nursing: Advancing of Humanistic Imperative. Fourth Edition. Lippincott Williams and Wilkins, Philadelphia, PA.

Tanghe A, Evers G, Paridaens R (1998). Nurses' assessments of symptom occurrence and symptom distress in chemotherapy patients. European Journal of Oncology Nursing 2(1):14-26.

Weingart S, Price J, Duncombe D, Connor M, Sommer K, Conley K, Bierer B, Ponte P (2007). Patient-reported safety and quality of care in outpatient oncology. Joint Commission on Quality and Patient Safety. 33:83-94. 
Wells M, Donnan PT, Sharp L, Ackland C, Fletcher J, Dewar JA (2008). A study to evaluate nurse-led on treatment review for patients undergoing radiotherapy for head and neck cancer. Journal of Clinical Nursing. 17(11):1428-39

Willard C, Luker K. (2007). Working with the team: strategies employed by hospital cancer nurse specialists to implement their role. Journal of Clinical Nursing. 16: 716724.

Wiseman T, Verity R, Ream E, Alderman E, Richardson A. (2005). Exploring the work of nurses who administer chemotherapy: A multi-methods study. London: King's College London.

World Medical Association (2016). WMA Declaration of Helsinki - Ethical Principles for Medical Research Involving Human Subjects.

http://www.wma.net/en/30publications/10policies/b3/ [Last accessed 25.07.2016. 